\title{
Replication and transcription of human papillomavirus type 58 genome in Saccharomyces cerevisiae
}

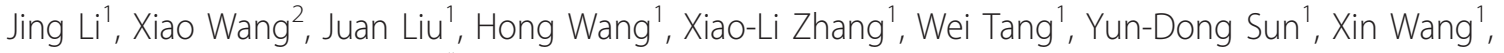
Xiu-Ping Yu', Wei-Ming Zhao ${ }^{1 *}$

\begin{abstract}
Background: To establish a convenient system for the study of human papillomavirus (HPV), we inserted a Saccharomyces cerevisiae selectable marker, Ura, into HPV58 genome and transformed it into yeast.

Results: HPV58 genome could replicate extrachromosomally in yeast, with transcription of its early and late genes. However, with mutation of the viral E2 gene, HPV58 genome lost its mitotic stability, and the transcription levels of E6 and E7 genes were upregulated.

Conclusions: E2 protein could participate in viral genome maintenance, replication and transcription regulation. This yeast model could be used for the study of certain aspects of HPV life cycle.
\end{abstract}

\section{Background}

Human papillomaviruses are small circular DNA viruses that infect epithelial cells and normally replicate as nuclear plasmids. The life cycle of papillomavirus is tightly linked to epithelial differentiation [1]. Among the high-risk HPV types associated with cervical cancer, human papillomavirus type 58 (HPV58) plays a more prominent role in Asian countries. HPV58 has been found in $5.9 \%$ of cervical cancer patients in China [2], with an unusually high prevalence in cervical cancer patients in specific areas of China: 33.3\% in Hong Kong [3] and $16.3 \%$ in Shanghai [4]. Despite the availability for biological study of few cell lines containing the DNA of high-risk HPVs such as 16, 18 and 31, no cell lines or animal models containing HPV58 have been established.

Saccharomyces cerevisiae is a species of budding yeast. The cellular mechanism required for DNA replication in S. cerevisiae is similar to that in human cells [5]. Studies have shown yeast to be a versatile organism for the study of viruses. Many types of DNA and RNA viruses, including HPVs, can directly replicate in yeast [6].

\footnotetext{
* Correspondence: zhaowm@sdu.edu.cn

'Department of Medical Microbiology, Shandong University School of

Medicine, Jinan, Shandong, 250012, PR China

Full list of author information is available at the end of the article
}

Although HPV6, 16 and 31 can replicate stably in yeast cells as nuclear plasmids $[7,8]$, whether HPV58 genome can replicate stably in yeast and whether the viral genes can be transcribed in yeast are unknown.

In the present study, we first explored the replication and transcription of HPV58 genome in the yeast system and investigated the function of E2 on vrial DNA replication and transcription. We found that HPV58 genome could replicate stably as an episome, with transcription of its early and late genes in yeast. However, with mutation of the E2 gene, HPV58 genome lost its mitotic stability and the transcription levels of E6 and E7 genes were upregulated. Thus, E2 protein can facilitate the replication and maintenance of HPV58 DNA and regulate viral gene transcription in yeast cells.

\section{Methods \\ DNA construction \\ HPV58-Ura}

The Ura gene was amplified from S. cerevisiae plasmid pRS316 with the sense (5'-GATCCACCGGTGGCAGATTGTACTGAGAGTG-3') and anti-sense (5'-CTAGCACCG GTGTAGTATACATGCATTTAC-3') primers containing the SgrA I site (underlined). The PCR-produced Ura was subcloned into the L2 open reading frame (ORF) of pEGFPN1-HPV58 to construct a
C Biomed Central

() 2010 Li et al; licensee BioMed Central Ltd. This is an Open Access article distributed under the terms of the Creative Commons Attribution License (http://creativecommons.org/licenses/by/2.0), which permits unrestricted use, distribution, and reproduction in any medium, provided the original work is properly cited. 
pEGFPN1-HPV58-Ura plasmid. The HPV58-Ura was released from pEGFPN1-HPV58-Ura by $B g l$ II digestion and recircularized by T4 DNA ligase for yeast transformation.

\section{HPV58-Ura-E2 mutant (HPV58-Ura-E2mt)}

A 1236 bp cassette was PCR amplified from pEGFPN1HPV58 with the sense (5'-CCACCAGGTGTAATGATGATTGGTAGCATC AAAGAC-3') and anti-sense (5'CATACCACCATGTGCAGAACCA-3') primers containing the Dra III site (underlined) and three stop codons (bold). The cassette was then substituted for the Dra III digested fragment (2924-4145 bp in HPV58 genome) in the pEGFPN1-HPV58-Ura plasmid to construct a pEGFPN1-HPV58-Ura-E2mt plasmid with three stop codons (2927-2932 bp) in the E2 ORF (2753-3829 bp). The HPV58-Ura-E2mt was released by Bgl II and recircularized for transformation.

\section{pDBLeu-E2}

The E2 protein expression plasmid was constructed as follows: E2 ORF was PCR amplified with the sense primer (5'-CCAAGCTTGAAAATTGGAAATCCT-3') containing the Hind III site (underlined) and anti-sense primer (5'-CTGCTAGCTTACAAGT CTTCTTCAGAGATCAACTTCTGTTCCAATGACATAACACCAGTACT-3') containing the Nhe I site (underlined) and a cMyc tag (bold). The E2 PCR product was subcloned into pDBLeu to construct the pDBLeu-E2.

\section{Yeast transformation}

S. cerevisiae strain W303-1B (MATa leu2-3 leu2-112 trp1-1 ura3-1 his3-11 his3-15 ade2-1 can1-100) were transformed with different sets of plasmids: 1). pRS316; 2). HPV58-Ura; 3). HPV58-Ura-E2mt; 4). HPV58-UraE2mt and pDBLeu (HPV58-Ura-E2mt/pDBLeu); 5). HPV58-Ura-E2mt and pDBLeu-E2 (HPV58-Ura-E2mt/ pDBLeu-E2). The transformed yeast were spotted on selective medium plates for 3-5 days. Single colonies were selected and cultured in yeast extract/peptone/dextrose (YPD) or synthetic complete (SC) dropout media (Clontech) for further analysis.

\section{Quantitative PCR (qPCR)}

Yeast were cultured in $10 \mathrm{ml}$ selective medium to an OD600 of 1.0 and yeast DNA was isolated as described [7]. The DNA was analysed by absolute qPCR with primers specific for E1 (sense: 5'-CTGCAATGGATGACCCTGAAG-3'; anti-sense: 5'-CCACTATCGTCTGCTGTTTCGT-3', amplicon: 136 bp, 878-1013 bp). Yeast 18S rDNA was used as internal control (sense: 5'TTGTGCTGGCGATGGTTCA-3'; anti-sense: 5'-TGCTGCCTTCCTTGGATGTG-3', amplicon: 152 bp). A standard curve was generated by amplification of a serial dilution of pEGFPN1-HPV58-Ura.

\section{Southern blot analysis}

Yeast harboring HPV58-Ura and HPV58-Ura-E2mt/ pDBLeu-E2 were grown in 25 -ml selective medium overnight to yield an OD600 of 1.0. HPV58-Ura-E2mt transformed yeast were cultured in $25 \mathrm{ml}$ selective medium for 3 days to yield an OD600 of 0.2, because of the poor growth in selective medium. Yeast DNA was isolated and digested with Xho I (no cut on HPV58 genome), Bgl II (1 cut), Hpa I (1 cut) or Dpn I (9 cuts) for $24 \mathrm{hr} . D p n$ I can digest methylated DNA isolated from bacteria only. The DNA was electrophoresed, blotted onto nylon membrane (Roche) and probed with an L1 specific mRNA probe labeled with digoxigenin by in vitro transcription according to the manufacturer's instructions (DIG RNA Labeling Kit, Roche).

\section{Western blot analysis}

Yeast protein was prepared from yeast harboring pDBLeu-E2, pDBLeu, and untransformed yeast as previously described [9] Protein samples were separated by SDS-PAGE electrophoresis and transferred to nitrocellulose membrane. Then the membrane was blocked with 3\% BSA in PBST and immunoblotted with antibody against cMyc (Santa Cruz). Membrane was washed and incubated with HRP conjugated secondary antibody. Chemilucent ECL Detection System (Millipore) was used to detect the signals according to the manufacturer's instruction.

\section{HPV58 genome stability assay}

The DNA stability assay was performed as previously described [10]. Transformed yeast were first grown in selective medium to mid-log phase and diluted to an OD600 of 0.1 in new cultures containing non-selective medium. The cultures were grown for $17 \mathrm{hr}$ (10 cell generations). The cultures at either 0 or 10 generations were diluted to an OD600 of 0.1. Aliquots of $5 \mu \mathrm{l}$ were spotted to selective and non-selective media. After 3 days of growth, the percentage of colonies containing viral DNA was determined by the ratio of the number of colonies on selective medium to those on non-selective medium. The percentage of DNA loss per cell generation was calculated by subtracting the percentage DNA retained after 10 generations from that at 0 generation and divided by the total number of generations.

\section{RNA extraction, RT-PCR and quantitative RT-PCR (qRT- PCR)}

Yeast were cultured in $10 \mathrm{ml}$ selective medium to an OD600 of 1.0 or 0.2 (HPV58-Ura-E2mt transformed yeast). Yeast DNA and RNA were isolated from the same samples. Yeast total RNA was isolated as previously described [11] and digested by DNase I 
(Fermentas) to remove the contaminating DNA. PCR involved use of DNase I-treated RNA as a template to ensure the complete digestion of contaminating DNA in RNA samples.

The DNase I treated RNA was analysed by RT-PCR and absolute qRT-PCR with primers specific for E1 (sense: 5'-CTGCAATGGATGACCCTGAAG-3'; antisense: 5'-CCACTATCGTCTGCTGTTTCGT-3', amplicon: 136 bp, 878-1013 bp), E2 (sense: 5'-GACAAAGCGACGACGACT-3'; anti-sense: 5'-GTCGTTGTGTT TCCGTTGT-3', amplicon: 335 bp, 3427- 3761 bp), E6 (sense: 5'-ACTATGTTCCAGGACGCAGAG-3'; antisense: 5'-ACCTCAGATCGCTGCAAAG-3', amplicon: 128 bp, 107-234 bp), E7 (sense: 5'-GACGAGGATGAAATAGGCTTG-3'; anti-sense:5'-CGTCGGTTG TTGTACTGTTGA-3', amplicon: 133 bp, 670-802 bp), L1 (sense: 5'-CTTGAAATAGGTAGGGGACAG-3'; anti-sense: 5'-CAATGGAGGACAATCAGTAGC-3', amplicon: $249 \mathrm{bp}, 5958-6206 \mathrm{bp}$ ) and L2 (sense: 5'CATAGTGACATATCGCCTGCTC-3'; anti-sense: 5'AGCCCCTATTTGCT TTCCAC-3', amplicon: 153 bp, 5051-5203 bp) respectively. Standard curves were generated by amplification of a serial dilution of pEGFPN1HPV58-Ura.

Relative qRT-PCR was ued to compare the transcription levels of E6 and E7 genes with or without E2 protein. Because HPV58 genomes have different replication efficiency in HPV58-Ura-, HPV58-Ura-E2mt- and HPV58-Ura-E2mt/pDBLeuE2-transformed yeast, we first compared the relative replication levels of the HPV58 genome in different transformants by qPCR. The E1 primers described previously and $18 \mathrm{~S}$ rDNA primers (sense:5'-TTGTGCTGGCGATGGTTCA-3'; anti-sense: 5'-TGCTGCCTTCCTTGGATGTG -3', amplicon: 152 $\mathrm{bp}$, as internal control) were used in qPCR. Then qRTPCR was performed to analyze the relative transcription levels of E6 and E7 genes of HPV58-Ura, HPV58-UraE2mt and HPV58-Ura-E2mt/pDBLeu-E2 in yeast, with the $18 \mathrm{~S}$ rRNA as internal control. The value of relative transcription levels was divided by the value of DNA relative replication levels to standardize the transcription templates.

\section{Results}

\section{Episomal replication of HPV58 in yeast}

Viral DNA copy number was detected by qPCR from five continuous yeast passages as shown in Table 1. The viral DNA copy number in per microliter of yeast DNA is relatively consistent in the five continous passages. Averagely, there are 3-5 copies of viral DNA in per yeast cell.

Southern blot was performed to investigate the replication form of HPV58 genome in yeast. As shown in Figure $1 \mathrm{~A}$ and $1 \mathrm{~B}$, when yeast DNA was treated with
Table 1 HPV58 genome copy number in cotinuous 5 passages:

\begin{tabular}{cc}
\hline Passage & viral DNA copies/ $\boldsymbol{\mu g}$ yeast DNA* \\
\hline P6 & $5.13 \times 10^{7} \pm 4.85 \times 10^{6}$ \\
P7 & $4.09 \times 10^{7} \pm 3.38 \times 10^{6}$ \\
P8 & $6.35 \times 10^{7} \pm 4.45 \times 10^{6}$ \\
P9 & $5.93 \times 10^{7} \pm 2.14 \times 10^{6}$ \\
P10 & $7.09 \times 10^{7} \pm 4.67 \times 10^{6}$ \\
\hline
\end{tabular}

DNA was isolated from yeast culture from passage 5 to passage 10. Viral DNA copies were quantitated by quantative PCR. The standard curve was generated by serially diluted pEGFPN1-HPV58-Ura. There is 3-5 copies of viral DNA in per yeast cells.

*Values are mean \pm standard deviation.

Dpn I or restriction enzymes which have no recognition site in HPV58 genome, the main form of HPV58 genome was open circular $(\mathrm{OC})$. The vortex process during yeast DNA isolation may disrupt the supercoiled plasmid (SC) and lead to the formation of OC plasmid. Yeast DNA digested by a single-cut restriction enzyme revealed only a single band representing the linear form of HPV58 (Figure 1A). Therefore, HPV58 genome could replicate episomally in yeast.

Furthermore, no band was detected in the $D p n$ I treated DNA isolated from HPV58-Ura-E2mt transformed yeast (Figure 1B, lane 1), which indicates that the E2 gene mutation induced instability and decreased the replication level of viral DNA. Transformation of pDBLeu-E2 into yeast restored the mitotic stability of HPV58-Ura-E2mt and clear bands were detected (Figure $1 \mathrm{~B}$, lane 2-6). We have tried to detect and compare the expression levels of E2 protein from HPV58-Ura and pDBLeu-E2 with anti-HPV16 E2 antibody, but no bands were detected. The transcription levels of E2 from HPV58-Ura and pDBLeu-E2 were compared with qRTPCR, the mRNA level of E2 from pDBLeu-E2 is 323 folds to that from HPV58-Ura (Figure 1C). Furthermore, we detected E2 protein in pDBLeu-E2 transformed yeast with anti-cMyc antibody as shown in Figure 1D. Therefore, E2 protein could facilitate viral genome replication and maintenance.

Role of E2 protein in maintaining HPV58 genome in yeast DNA stability assay was performed to investigate the maintenance function of E2 protein on HPV58 genome. The DNA loss per cell generation for HPV58-Ura (1.9\%) was comparable to that of the yeast plasmid pRS316 (2.1\%) which contains centromeric elements (CEN) (Figure 2). Thus, the HPV58 genome was as stable as a yeast CEN containing plasmid.

Yeast harboring HPV58-Ura-E2mt grew poorly in selective medium, with no colony generated on the selective plates at G0 and G10. However, with transformation of pDBLeu-E2 and re-expression of E2 protein 


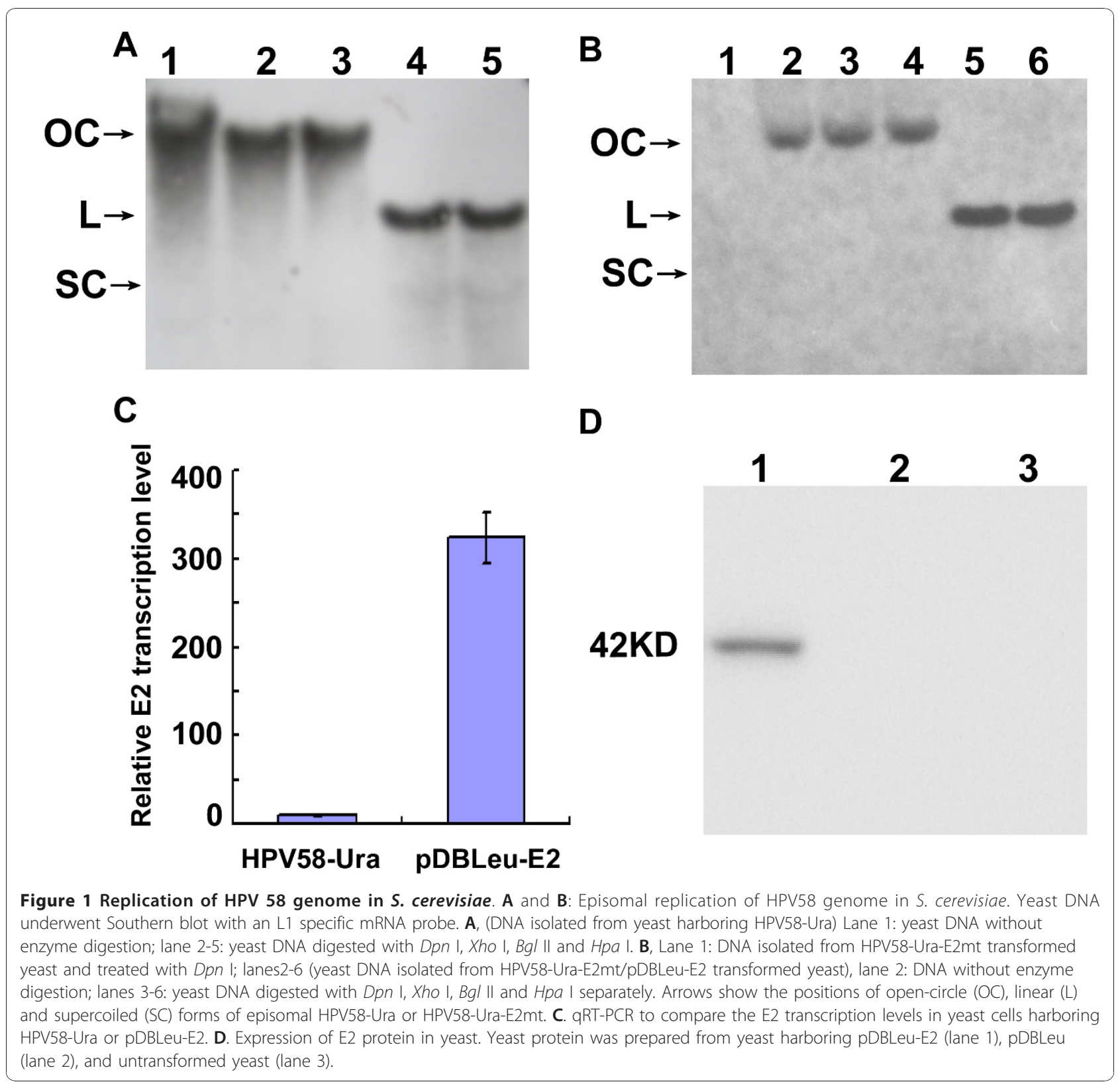

(Figure 1C, D), the HPV58 genome restored its mitotic stability to a DNA loss rate of $2.5 \%$ per cell generation (Figure 2). Therefore, E2 protein is critical to the mitotic stability of the HPV58 genome in yeast.

\section{Suppression of E6 and E7 transcription by E2 protein in yeast}

RT-PCR analysis revealed the transcription of both early (E1, E2, E6, E7) and late genes (L1 and L2) of HPV58Ura in yeast (Figure 3A). To further investigate the transcription levels of viral genes in yeast cells, qRT-PCR was performed separately with gene specific primers. The results revealed that HPV58 early and late genes have different transcription efficiency (Table 2).
To explore the regulatory function of E2 protein on viral gene transcription, E6 and E7 genes transcription levels in the three yeast transformants were compared by qRT-PCR. We first compared the DNA relative replication levels of HPV58 genome in yeast by qPCR. With the relative replication level of HPV58-Ura set to 1.0, DNA relative replication levels were 0.03 for HPV58-Ura-E2mt and 0.54 for HPV58-Ura-E2mt/pDBLeu-E2 (Figure 3B).

Then qRT-PCR was performed to compare the transcription levels of E6 and E7 genes. Because wild type HPV58 genome and E2 mutant HPV58 genome have different replication levels, the relative transcription levels were divided by the value of relative replication levels. With E6 and E7 transcripion levels of the 


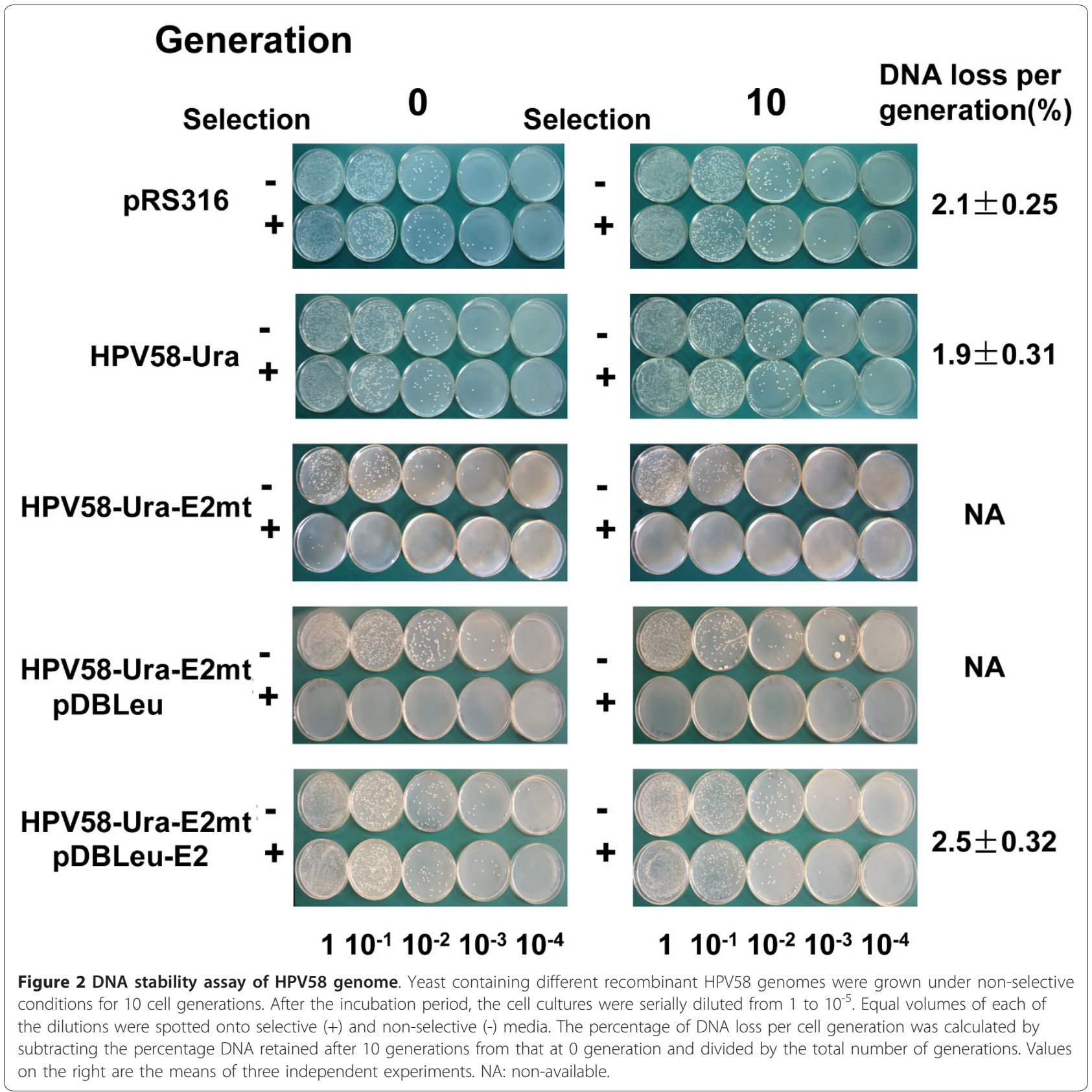

HPV58-Ura transformants set to 1.0, the relative transcription levels of E6 were 8.62 for HPV58-Ura-E2mt and 1.02 for HPV58-Ura-E2mt/pDBLeu-E2. The E7 relative transcription levels were 1.96 for HPV58-Ura-E2mt and 0.74 for HPV58-Ura-E2mt/pDBLeu-E2 (Figure 3C). Therefore, E2 protein could suppress the E6 and E7 genes transcription levels when HPV58 genome persists episomally in yeast.

\section{Discussion}

Previous reports have shown that HPV1, 6, 11, 16, 18 and 31 can replicate their genomes as episomal plasmids in yeast. The genomes of HPV6, 16 and 31 are mitotically stable in yeast, however HPV1, 11, and 18 are unstable in yeast $[7,8,12]$. In the present study, we showed that HPV58 genome, consistent with the HPV6, 16 and 31, can replicate stably as an episomal plasmid in yeast.

Stable maintenance through replication of extrachromosomal DNA in yeast requires autonomous replication sequences (ARS) $[13,14]$, and centromeric elements (CEN) [15]. There is a 10 of 11 nucleotide match to the consensus core ARS sequence (CAS) (5'-[A/T]TTTAT [A/G]TTT[A/T]-3') in the HPV58 genome (accession 


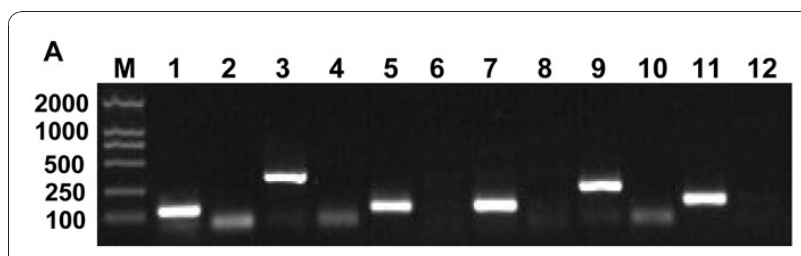

B

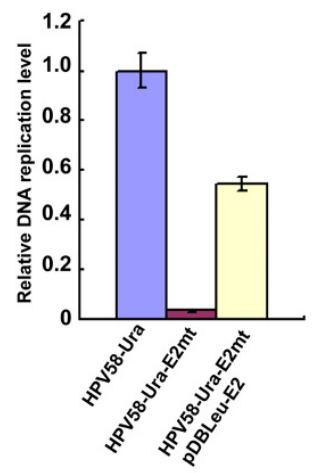

C

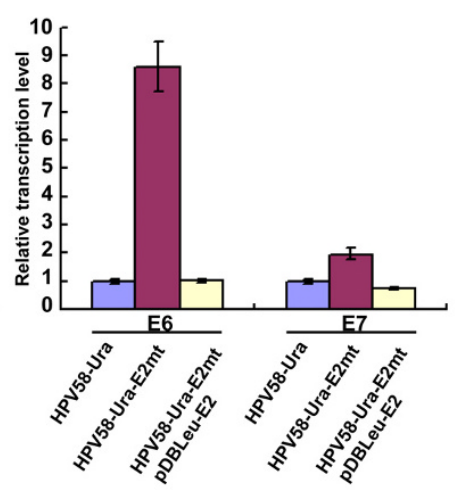

Figure 3 Transcription of HPV58 genes in S. cerevisiae. A. RTPCR analysis of transcriptional expression of HPV58 early and late genes in the HPV58-Ura transformed yeast. E1 (lanes 1, 2), E2 (lanes 3, 4), E6 (lanes 5, 6), E7 (lanes 7, 8), L1 (lanes 9, 10) and L2 (lanes 11, 12) from the CDNA samples. Total RNA was isolated from HPV58-Ura transformed yeast and digested with DNase I. The DNase I treated RNA was amplified to ensure the complete digestion of contaminating viral DNA (lanes 2, 4, 6, 8, 10 and 12). DNase I completely digested RNA were then analysized by RT-PCR (lanes 1 , 3, 5, 7, 9 and 11). B. Relative replication levels of HPV58 genomes in HPV58-Ura, HPV58-Ura-E2mt and HPV58-Ura-E2mt/pDBLeu-E2 transformed yeast. $18 \mathrm{~S}$ rDNA was used as internal control. The DNA relative replication levels of HPV58-Ura-E2mt (0.03) and HPV58-UraE2mt/pDBLeu-E2 (0.54) are relative to that of HPV58-Ura, which was set to 1.0. Standard deviations are indicated by error bars. C. Relative transcription levels of E6 and E7 genes for HPV58-Ura, HPV58-Ura-E2mt and HPV58-Ura-E2mt/pDBLeu-E2. DNase I treated RNA was analysized by qRT-PCR with $18 \mathrm{~S}$ rRNA as internal control. As for HPV58 genomes have different replication efficiency in the presence or absence of E2 protein (Figure 3B), the transcription levels of E6 and E7 genes were then standardized to the relative DNA replication assay. The E6 and E7 genes transcriptional levels of HPV58-Ura were set to 1.0. The transcription levels of E6 are 8.62 for HPV58-Ura-E2mt and 1.02 for HPV58-Ura-E2mt/pDBLeu-E2. The E7 transcription levels are 1.96 for HPV58-Ura-E2mt and 0.74 for HPV58Ura-E2mt/pDB Leu-E2 relative to that of HPV58-Ura, which was set to 1.0. Standard deviations are indicated by error bars.

no. D90400) at nucleotides 1225 to 1216 . Yeast origin recognition complex (ORC) may bind to the CAS-like element and initiate viral genome replication. It has generally been thought that replication of papillomaviruses is dependent upon the presence of E1, a DNA helicase, and E2, a transcription and maintenance factor $[16,17]$. $\mathrm{E} 1$ and $\mathrm{E} 2$ protein, perhaps in conjunction with CASlike elements, conduct the replication of HPV58 genome in yeast.
Table 2 Transcription levels of HPV58 genes in yeast

\begin{tabular}{cc}
\hline Gene & mRNA Copies/ $\boldsymbol{\mu g}$ total RNA* \\
\hline E1 & $6.54 \times 10^{5} \pm 1.03 \times 10^{4}$ \\
E2 & $2.42 \times 10^{4} \pm 1.83 \times 10^{3}$ \\
E6 & $2.48 \times 10^{4} \pm 1.77 \times 10^{3}$ \\
E7 & $3.75 \times 10^{5} \pm 2.68 \times 10^{4}$ \\
L1 & $2.08 \times 10^{5} \pm 1.73 \times 10^{4}$ \\
L2 & $1.34 \times 10^{6} \pm 1.24 \times 10^{4}$
\end{tabular}

Note: Total RNA isolated from yeast cells were digested with DNase I and converted into CDNAs by reverse transcription. The standard curve was generated by serially diluted pEGFPN1-HPV58-Ura. The levels of the viral gene transcripts examined in per microgram total RNA were analyzed by qRT-PCR.

*Values are mean \pm standard deviation.

Replication of genomes in yeast is a common feature of HPVs. Different HPV types showed different genome stability in yeast cells. Two kinds of mechanisms may control the mitotic stability of the HPV58 genomes in yeast. First, HPV58 sequences may contain cis-elements that can substitute for the CEN required for the maintenance of extrochromosomal DNA in S. cerevisiae. The second mechanism may be the function of HPV58 E2 protein, a maintenance protein that can tether viral genomes to mitotic chromosomes in dividing cells $[18,19]$. Angeletti et al. (2002) reported that the HPV16 genome can replicate stably in yeast cells with the E2 gene interrupted by introducing stop codons. Kim et al (2005) also identified the CEN-like cis-elements in the HPV16 genome in yeast and mammalian cells and concluded that the maintenance of the HPV16 genome depends on the CEN-like cis-elements, perhaps in conjunction with E2 protein. HPV16 can maintain the mitotic stability of its genome in an E2-independent manner in yeast and mammalian cells $[7,20,21]$.

In contrast to HPV16 genome, the maintenance of HPV58 genome depends on E2 protein. The wild-type HPV58 genome can replicate stably in transformed yeast cells. However, with the HPV58 E2 gene interrupted by mutation, HPV58-Ura-E2mt genome was unstable in yeast. Introduction of pDBLeu-E2 and reexpression of E2 protein restored the stability of HPV58-Ura-E2mt genome in yeast cells. Therefore, the maintenance and faithful segregation of HPV58 genome depends on E2 protein. The HPV58 genome has no CEN-like cis-elements. This specific maintenance pattern of the HPV58 genome may provide a possible way to interfere with the early stage of HPV58 infection by blocking the expression of E2 protein.

Previous reports of the HPV/yeast system focused on the replication of HPV DNA. In fact, the HPV/yeast system could be a model for the study of HPV gene transcription. Although we failed to detect the mRNA of HPV58 early and late genes by Northern blot, which may be due to the low transcription levels of viral genes 
in yeast. RT-PCR results revealed the transcription of HPV58 early and late genes in yeast. Moreover, qRTPCR analysis of transcription regulation function of E2 protein revealed both E6 and E7 mRNA levels were upregulated with HPV58-Ura-E2mt introduced into yeast. However, with re-expression of E2 protein, the mRNA levels were downregulated. These results confirm that E2 protein can suppress E6 and E7 genes transcription when HPV58 genome persists episomally in yeast.

Bechold et al (2003) reported that E2 protein had no affect on E6/E7 expression in mammalial cells [22]. Bouvard et al (1994) reported that HPV16 and 18 E2 protein could activate their early promoter in CAT luciferase reporter plasmid. However, overexpression of E2 repressesd the early promoter [23]. These reports are different from our data that E2 protein repressed early promoter transcription in yeast. The reasons should be the different cell lines used, different expression levels of E2 protein and different conformation of viral minichromosomes in cells, especially the chromatin conformation of long control region (LCR).

The early promoter of HPV locates in the LCR of the viral genome [24]. The LCR has four E2 protein binding sites (E2BS). E2BS4 is far from the TATA box. The other three E2BS (E2BS1, E2BS2 and E2BS3) are proximal to the TATA box. E2 protein binding to E2BS4, which is far from TATA box, stimulates the transcription level of E6 and E7. However, binding of E2 protein to the other three E2BS represses transcription through steric hindrance of the interaction with the transcriptional initiation factor TFIID at the proximal TATA box $[25,26]$. Binding of E2 protein to a specific E2BS is determined by the genome status. HPV58 E2 protein might interact with the E2BS proximal to the TATA box when the HPV58 genome is maintained as an episomal plasmid in yeast.

\section{Conclusions}

The HPV58/yeast system we used was able to direct the stable replication of the HPV58 genome and induce the transcription of the early and late genes. As compared with maintenance of the HPV16 genome, HPV58 genome strictly depends on E2 protein. E2 protein can supress the transcription of E6 and E7 genes. With its ease of handling and similarity to mammalian system, the yeast system we described is useful for future study of HPV58 genome replication and the regulatory mechanism of viral gene transcription.

\section{List of abbreviations}

ARS: autonomously replicating sequences; CEN: centromeric elements; HPV: Human papillomavirus; LCR: long control region; qPCR: quantitative PCR; QRT-PCR: quantitative RT-PCR; RT-PCR: reverse transcription PCR;

\section{Competing interests}

The authors declare that they have no competing interests.

\section{Authors' contributions}

$J$ constructed the HPV58-Ura-E2mt, pDBLeu-E2, participated in Sountern blot, Northern blot, Western blot, DNA stability assay and drafted the manuscript. XW constructed HPV58-Ura, participated in Western blot, DNA stability assay and PCR. JL and HW participated in Southern blot and DNA stability assay. XLZ participated in yeast transformation and DNA stability assay. WT and YDS helped to perform the real time quantitative PCR, Southern blot and Northern blot. XW participated in primers and probe design. XPY gave advices on the design of this study. WMZ designed the study and drafted the manuscript. All authors read and approved the final manuscript.

\section{Acknowledgements}

This work was funded by the National Natural Science Foundation of China (30470086 to WMZ). We thank Dr. Kong-Nan Zhao (University of Queensland Centre for Clinical Research, Australia) and Dr. Peter Angeletti (University of Nebraska-Lincoln School of Life Science) for their quidance on the experiments.

\section{Author details}

'Department of Medical Microbiology, Shandong University School of Medicine, Jinan, Shandong, 250012, PR China. 'Department of Pathology, Shandong University School of Medicine, Jinan, Shandong, 250012, PR China.

Received: 25 July 2010 Accepted: 15 December 2010

Published: 15 December 2010

\section{References}

1. Doorbar J: The papillomavirus life cycle. J Clin Virol 2005, 32:7-15.

2. Wu Y, Chen Y, Li L, Yu G, Zhang Y, He Y: Associations of high-risk HPV types and viral load with cervical cancer in China. J Clin Virol 2005, 35:264-269.

3. Chan PK, Cheung TH, Li WW, Lo KW, Chan MY, Cheung JL, Cheng AF: Association of Human Papillomavirus Type 58 Variant With the Risk of Cervical Cancer. J Natl Cancer Inst 2002, 94:1249-1253.

4. Liaw KL, Hsing AW, Schiffman MH, You SL, Zhang T, Burk R, Chen CJ: Human papillomavirus types 52 and 58 are prevalent in cervical cancers from Chinese women. Int J Cancer 1997, 73:775-776.

5. Bielinsky AK, Gerbi SA: Where it all starts: eukaryotic origins of DNA replication. J Cell Sci 2001, 114:643-651.

6. Alves-Rodrigues I, Galão RP, Meyerhans A, Díez J: Saccharomyces cerevisiae: A useful model host to study fundamental biology of viral replication. Virus Research 2006, 120:49-56.

7. Angeletti PC, Kim K, Fernandez FJ, Lambert PF: Stable replication of papillomavirus genomes in Saccharomyces cerevisiae. J Virol 2002, 76:3350-3358.

8. Chattopadhyay A, Schmidt MC, Khan SA: Identification of a 450-bp region of human papillomavirus type 1 that promotes episomal replication in Saccharomyces cerevisiae. Virology 2005, 340:133-142.

9. Kushinirov W: Rapid and reliable protein extraction from yeast. Yeast 2000, 16:857-860.

10. Kapoor P, Shire K, Frappier L: Reconstitution of Epstein-Barr virus-based plasmid partitioning in budding yeast. EMBO J 2001, 20:222-230.

11. Li J, Liu J, Wang X, Zhao L, Chen Q, Zhao WM: A water bath method for preparation of RNA from Saccharomyces cerevisiae. Analy Bioch 2009, 384:189-190.

12. Rogers AJ, Loggen $M$, Lee $K$, Angeletti PC: Varying efficiency of long term replication of papillomaviruses in Saccharomyces cerevisiae. Virology 2008, 381:6-10.

13. Hsiao CL, Carbon J: High-frequency transformation of yeast by plasmids containing the cloned yeast. ARG4 gene. Proc Natl Acad Sci 1979, 76:3829-3833.

14. Stinchcomb DT, Struhl K, Davis RW: Isolation and characterisation of a yeast chromosomal replicator. Nature 1979, 282:39-43.

15. Maraherns $Y$, Stillman B: A yeast chromosomal origin of DNA replication defined by multiple functional elements. Science 1992, 255:817-823. 
16. Rabson MS, Yee C, Yang YC, Howley PM: Bovine papillomavirus type $13^{\prime}$ early region transformation and plasmid maintenance functions. J Virol 1986, 60:626-634.

17. Ustav M, Ustav E, Szymanski P, Stenlund A: Identification of the origin of replication of bovine papillomavirus and characterization of the viral origin recognition factor E1. EMBO J 1991, 10:4321-4329.

18. Abroi A, llves I, Kivi S, Ustav M: Analysis of chromatin attachment and partitioning functions of bovine papillomavirus type 1 E2 protein. J Virol 2004, 78:2100-2113.

19. Lehman CW, King DS, Botchan MR: A papillomavirus E2 phosphorylation mutant exhibits normal transient replication and transcription but is defective in transformation and plasmid retention. J Virol 1997, 71:3652-3665.

20. Kim K, Angeletti PC, Hassebroek EC, Lambert PF: Identification of cis-Acting Elements That Mediate the Replication and Maintenance of Human Papillomavirus Type 16 genomes in Saccharomyces cerevisiae. J Virol 2005, 79:5933-5942.

21. Pittayakhajonwut D, Angeletti PC: Analysis of cis-elements that facilitate extrachromosomal persistence of human papillomavirus genomes. Virology 2008, 374:304-314

22. Bechtold V, Beard P, Raj K: Human Papillomavirus Type 16 E2 Protein Has No Effect on transcription from episomal viral DNA. J Virol 2003, 77:2012-2028

23. Bouvard V, Storey A, Pim D, Banks L: Characterization of the human papillomavirus E2 protein: evidence of trans-activation and transrepression in cervical keratinocytes. EMBO J 1994, 13:5451-5459.

24. Rosenstierne MW, Vinther J, Hansen CN, Prydsoe M, Norrild B: Identification and characterization of a cluster of transcription start sites located in the E6 ORF of human papillomavirus type 16. J Gen Virol 2003, 84:2909-2920.

25. Demeret C, Desaintes C, Yaniv M, Thierry F: Different mechanisms contribute to the E2-mediated transcriptional repression of human papillomavirus type18 viral oncogenes. J Virol 1997, 71:9343-9349.

26. Sanchez IE, Dellarole M, Gaston K, de Prat Gay G: Comprehensive comparison of the interaction of the E2 master regulator with its cognate target DNA sites in 73 human papillomavirus types by sequence statistics. Nucleic Acids Res 2008, 36:756-769.

\section{Submit your next manuscript to BioMed Central and take full advantage of:}

- Convenient online submission

- Thorough peer review

- No space constraints or color figure charges

- Immediate publication on acceptance

- Inclusion in PubMed, CAS, Scopus and Google Scholar

- Research which is freely available for redistribution

Submit your manuscript at www.biomedcentral.com/submit
Biomed Central 